\title{
Finsler Geodesics Evolution Model for Region based Active Contours
}

\section{Da Chen ${ }^{1}$}

chenda@ceremade.dauphine.fr

Jean-Marie Mirebeau ${ }^{2}$

jean-marie.mirebeau@math.u-psud.fr

Laurent D. Cohen ${ }^{1}$

cohen@ceremade.dauphine.fr
${ }^{1}$ CEREMADE, CNRS, University Paris Dauphine, PSL Research University, UMR 7534, 75016 PARIS, FRANCE

${ }^{2}$ Laboratoire de mathématiques d'Orsay, CNRS, Université Paris-Sud, Université Paris-Saclay, 91405 ORSAY, FRANCE
In this paper, we introduce a new deformable model for image segmentation, by reformulating a region based active contours energy into a geodesic contour energy involving a Finsler metric.

Let $\Omega \subset \mathbb{R}^{2}$ be the image domain and $\gamma:[0,1] \rightarrow \Omega$ be a regular curve with outward normal vector $\mathcal{N}$. Given a function $f: \Omega \rightarrow \mathbb{R}$ of interest, we consider the curve evolution scheme:

$$
\frac{\partial \gamma}{\partial \tau}=f(\gamma) \mathcal{N}
$$

where $\tau$ denotes time. This curve evolution equation can be regarded as a gradient descent, thus a minimization procedure [2], for the functional

$$
F(\gamma)=\int_{K} f(\mathbf{x}) d \mathbf{x},
$$

where $K \subset \Omega$ is the region inside the closed curve $\gamma:=\partial K$. A complete active contour energy with a curve length regularization can be defined as

$$
E(\gamma)=\alpha F(\gamma)+\int_{0}^{1} P(\gamma(t))\|\dot{\gamma}(t)\| d t,
$$

where $P$ is an edge based potential function, and $\alpha>0$ is a constant.

Reformulation as Finsler Geodesic Energy: Suppose $\mathcal{V}_{\perp}: \Omega \rightarrow \mathbb{R}^{2}$ to be a continuously differentiable vector field defined over the domain $\Omega$ such that $\mathcal{V}_{\perp}$ satisfies the following divergence equation:

$$
\nabla \cdot \mathcal{V}_{\perp}(\mathbf{x})=\alpha f(\mathbf{x}), \quad \forall \mathbf{x} \in \Omega,
$$

where $f$ is the first order derivative function used in $(2)$ and $\nabla \cdot \mathcal{V}_{\perp}(\mathbf{x})$ denotes the divergence value of a vector $\mathcal{V}_{\perp}(\mathbf{x})$. Letting $M$ be the counterclockwise rotation matrix with rotation angle $\theta=\pi / 2$, by divergence theorem, the regional energy $F$ in (2) can be expressed as

$$
\begin{aligned}
\alpha F(\gamma) & :=\alpha \int_{K} f(\mathbf{x}) d \mathbf{x}=\int_{K} \nabla \cdot \mathcal{V}_{\perp}(\mathbf{x}) d \mathbf{x} \\
& =\int_{0}^{1}\left\langle\mathcal{V}_{\perp}(\gamma(t)), \mathcal{N}(t)\right\rangle\|\dot{\gamma}(t)\| d t \\
& =\int_{0}^{1}\left\langle M^{\mathrm{T}} \mathcal{V}_{\perp}(\gamma(t)), M^{\mathrm{T}} \mathcal{N}(t)\|\dot{\gamma}(t)\|\right\rangle d t \\
& =\int_{0}^{1}\langle\mathcal{V}(\gamma(t)), \dot{\gamma}(t)\rangle d t
\end{aligned}
$$

where $\mathcal{V}=M^{\mathrm{T}} \mathcal{V}_{\perp}$. Unit vector $\mathcal{N}$ is the outward normal vector of contour $\gamma$ and $\dot{\gamma}$ is the tangent vector of $\gamma$ in clockwise order. Indeed, $\mathcal{T}=M^{\mathrm{T}} \mathcal{N}$ is the tangent vector and

$$
\dot{\gamma}(t)=\|\dot{\gamma}(t)\| \mathcal{T}(t)=\|\dot{\gamma}(t)\| M^{\mathrm{T}} \mathcal{N}(t), \quad \forall t \in[0,1] .
$$

One can introduce a Finsler metric $\mathcal{F}: \Omega \times \mathbb{R}^{2} \rightarrow \mathbb{R}$ :

$$
\mathcal{F}(\mathbf{x}, \mathbf{u})=P(\mathbf{x})\|\mathbf{u}\|+\langle\mathcal{V}(\mathbf{x}), \mathbf{u}\rangle,
$$

which is positive, provided one has the smallness condition [1]:

$$
\|\mathcal{V}(\mathbf{x})\|<P(\mathbf{x}), \quad \forall \mathbf{x} \in \Omega .
$$

In practice, it is difficult to satisfy the smallness condition (10). Assuming that $\forall \mathbf{x} \in \Omega, P(\mathbf{x}) \geq 1$, we make use of the following condition:

$$
\|\mathcal{V}(\mathbf{x})\|<\min _{\mathbf{y} \in \Omega}\{P(\mathbf{y})\}=1, \quad \forall \mathbf{x} \in \Omega .
$$

In view of $\mathcal{F}$ and (5), the energy $E$ (3) is converted to the Finsler geodesic energy:

$$
\mathcal{L}(\gamma)=\int_{0}^{1} \mathcal{F}(\gamma(t), \dot{\gamma}(t)) d t .
$$

Computing the vector field $\mathcal{V}_{\perp}$ and Finsler Metric $\mathcal{F}$ : The minimization procedure of $\mathcal{L}(12)$ is solved inside a neighbourhood $U$ instead of the whole domain $\Omega$. This means that we only require the vector field $\mathcal{V}^{\perp}$ defined over $U$. In order to satisfy the smallness condition (10), it is natural to select a solution to (4) minimizing an energy

$$
\min \left\{\int_{U}\left\|\mathcal{V}_{\perp}(\mathbf{x})\right\|^{2} d \mathbf{x}\right\}, \quad \text { s.t. } \quad \nabla \cdot \mathcal{V}_{\perp}(\mathbf{x})=\alpha f(\mathbf{x}), \quad \forall \mathbf{x} \in U .
$$

Despite the rich regularity for solutions to elliptic PDEs, we could not find a result directly implying that the solution to (13) obeys the desired smallness condition (10). However, such a result can easily be established for a different solution to the divergence equation (4), given by convolution with an explicit kernel

$$
\mathcal{V}_{\perp}(\mathbf{x})=\frac{\alpha}{2 \pi} \int_{U} \frac{\mathbf{x}-\mathbf{y}}{\|\mathbf{x}-\mathbf{y}\|^{2}} f(\mathbf{y}) d \mathbf{y} .
$$

In that case one indeed obtains using Holder's inequality

$$
\begin{aligned}
\frac{2 \pi}{\alpha}\left\|\mathcal{V}_{\perp}(\mathbf{x})\right\| & \leq\|f\|_{q}\left(\int_{U} \frac{1}{\|\mathbf{x}-\mathbf{y}\|^{p}} d \mathbf{y}\right)^{\frac{1}{p}} \\
& \leq\|f\|_{q}\left(\int_{D_{U}} \frac{1}{\|\mathbf{x}-\mathbf{z}\|^{p}} d \mathbf{z}\right)^{\frac{1}{p}}=\eta A_{U}^{\mu}\|f\|_{q},
\end{aligned}
$$

where $D_{U}$ is a disk centered around $\mathbf{x}$ and with the same area $A_{U}$ as $U$. $p, q$ are two positive constants obeying $1 / p+1 / q=1$ and $q>2$. $\eta$ is a constant and $\mu=\frac{1}{p}-\frac{1}{2}>0$. $\|f\|_{q}$ is the $L^{q}$ norm of $f$ on $U$. The condition (11) is thus satisfied when the area of $U$ is sufficiently small.

Finsler Metric Construction: The vector field $\mathcal{V}_{\perp}$ solution to (13) depends on the neighbourhood $U$. In order to obtain a vector field obeying $\left\|\mathcal{V}_{\perp}\right\|_{\infty}<1$, one choose a tubular neighbourhood $U$ with small width hence a small area. On the other hand, $U$ is regarded as the search space for the next evolutional curve. A small $U$ may therefore make the algorithm fall into undesirable local minimas of the geodesic energy $\mathcal{L}$. Thus we use a trick to solve this problem by invoking a non-linear mapping increasing function $T: \mathbb{R}^{+} \rightarrow(0,1)$ defined as $T(x)=1-\exp (-x), \forall x>0$. Thus the new vector field $\overline{\mathcal{V}}$ can be expressed by

$$
\overline{\mathcal{V}}(\mathbf{x})=T\left(\left\|\mathcal{V}_{\perp}(\mathbf{x})\right\|\right) M^{-1} \mathcal{V}_{\perp}(\mathbf{x}) /\left\|\mathcal{V}_{\perp}(\mathbf{x})\right\|, \quad \forall \mathbf{x} \in \Omega
$$

where the smallness condition (11) will be immediately satisfied and $M$ is the counter-clockwise rotation matrix with rotation angle $\theta=\pi / 2$. Based on the vector field $\overline{\mathcal{V}}$, the Finsler metric is denoted by $\overline{\mathcal{F}}$ and the geodesic energy $\overline{\mathcal{L}}$ is defined by (12) with $\mathcal{F}:=\overline{\mathcal{F}}$.

The minimization of $E(3)$ is transferred to the minimization of $\overline{\mathcal{L}}$. Note that since in general we induce $\overline{\mathcal{L}}$ with a nonlinear mapping $T$, there is in fact slight difference in the minimization problems and the results show that our geodesic method is very efficient and robust. The non-linear mapping $T$ is reasonable: 1) The minimization of $E$ in (5) is relevant to both the directions of $\gamma$ and the norm of $\mathcal{V}$, i.e., minimizing $E$ is to find a path $\gamma$, for which the direction $\gamma^{\prime}(t)$ for each $t \in[0,1]$ should be as opposite to $\mathcal{V}(\gamma(t))$ as possible and the norm $\|\mathcal{V}(\gamma(t))\|$ should be as large as possible, giving the relevance between the minimization problems of $E$ and $\overline{\mathcal{L}}$. Introducing $T$ will not modify both goals of the minimization problems. 2) When the Finsler geodesics evolution scheme tends to stabilize, one can reduce the width of tubular neighbourhood $U$. Thus $T(\|\mathcal{V}\|) \approx\|\mathcal{V}\|$ as $\|\mathcal{V}\|$ is small.

[1] Jean-Marie Mirebeau. Efficient fast marching with Finsler metrics. $\mathrm{Nu}$ merische Mathematik, 126(3):515-557, 2014.

[2] Song Chun Zhu and Alan Yuille. Region competition: Unifying snakes, region growing, and Bayes/MDL for multiband image segmentation. IEEE Trans. on Pattern Analysis and Machine Intelligence, 18(9):884-900, 1996. 